\title{
EVOLUTION WITHIN THE MATURITY CONCEPT OF BIM
}

\author{
PIERRE MARTIN, DJAOUED BELADJINE \& KARIM BEDDIAR \\ Ecole d'ingénieurs CESI, Laboratoire d'Innovation Numérique pour les Entreprise \\ et les Apprentissages au service de la Compétitivité des Territoires, France
}

\begin{abstract}
Many construction companies in France are rather shy today with regards to the adoption of BIM because the process is not sufficiently understood. Companies therefore need effective support to ensure their digital transition. The success of this transformation is strongly related to the level of maturity of the companies in the deployment of BIM. It is in this context that we decided to undertake research work trying to propose a model to companies to assess their level of maturity BIM. This process has been established with the goal of providing user the solutions specific to each context as well as adjustments at the level of actions. The evaluation process is based on critical success factors associated with the BIM concept of maturity and concrete actions. This will allow the user to target the primordial issues and to invest to achieve them in order to progress in BIM maturity levels. The model implemented is based on a cumulative type model such as the CMM, which is a reliable indicator of maturity. The criteria are linked to critical success factors that must be prioritized to improve the level of maturity. Each of these factors is linked to actions, best reflecting the realities of BIM's maturity levels. Adjustments were subsequently made concerning the number and relevance of the actions selected. In order to ensure the relevance of the established model, a survey was conducted among construction professionals. It appeared that most practices and expectations of companies surveyed revolved around technical issues and this was for companies of the VSE/SME type. The highest expectations were found to have better cost analysis and optimization of the construction schedule. These results were subsequently incorporated into the model, which is still being tested. The model defined is committed to comprehensively accompany the user in the validation of maturity levels.

Keywords: BIM, BIM maturity, BIM management, BIM maturity model.
\end{abstract}

\section{INTRODUCTION}

Further to these technical considerations, the Building Information Model defends a project management style and its own building life cycle philosophy. The transition to the BIM is now initiated, and some precursors already harvests the full benefits. However, the radical changes generated by its implementation are far from being able to be supported by all the players in the construction industry. A large part of them encounter difficulties to understand and to use BIM correctly or exclude the possibility of integrating it definitively.

In anticipation to the modification reference model context, scientific research strives to characterize the potential benefits of BIM, its technological developments stakes, and its impact on the business processes companies. However, it is struggling to provide sufficient, persuasive and profitable support to construction professionals [1], [2]. The rapid and recent emergence of issues linked with BIM, has not given enough time for researches to deepen their studies. This analysis could be made thanks to a literary review carried out on the subject before the achievement of our study. Starting from identified gaps, the choice to focus on the challenges of increasing proficiency in the BIM maturity levels has been made. These are lacking structured conceptual approach, except for the existing maturity models. The objectives are then, to provide concrete assistance to companies willing to engage in BIM maturity levels by using all the information collected [3], [4]. This study is the opportunity to make links between different maturity models, from critical success factors to practical actions to implement in a company wishing to move toward the maturity levels of BIM [5], [6] BIM abroad and in France. 


\section{DEVELOPMENT OF MATURITY IN BIM}

The implementation of BIM refers to a complete integration of itself. It ensures the full potential within the supply chain and the various organizations in the constructions industry [7], [8].

BIM is implemented in construction companies on the basis of empirical data operating in specific business cases without considering the need to change practices in order to make them more efficient. The challenge of implementing BIM to ensure its optimal deployment lies in challenging and redesigning organizational, institutional and contractual practices [9], [10].

In order to meet this type of challenge, the approach by analyzing the maturity of the company is essential in order to facilitate the management of organizational change for the implementation of an information technology such as BIM [11], [12].

\subsection{Maturity concept}

This concept proposes to qualify or/and quantify the development of a subject. It offers the potential to measure the acquisition of own capacities in terms of benchmarks to stagger and quantify this development. This evaluation identifies the company's weaknesses or shortcomings and thus targets the capacities to reach the desired level [13], [14].

\subsection{BIM maturity levels}

BIM maturity levels represent the degree of maturity of the collaborative process between the various construction stakeholders. Fig. 1 below presents the transition from isolated work (Levels 0 and 1) to collaborative work (Levels 2 and 3) [15].

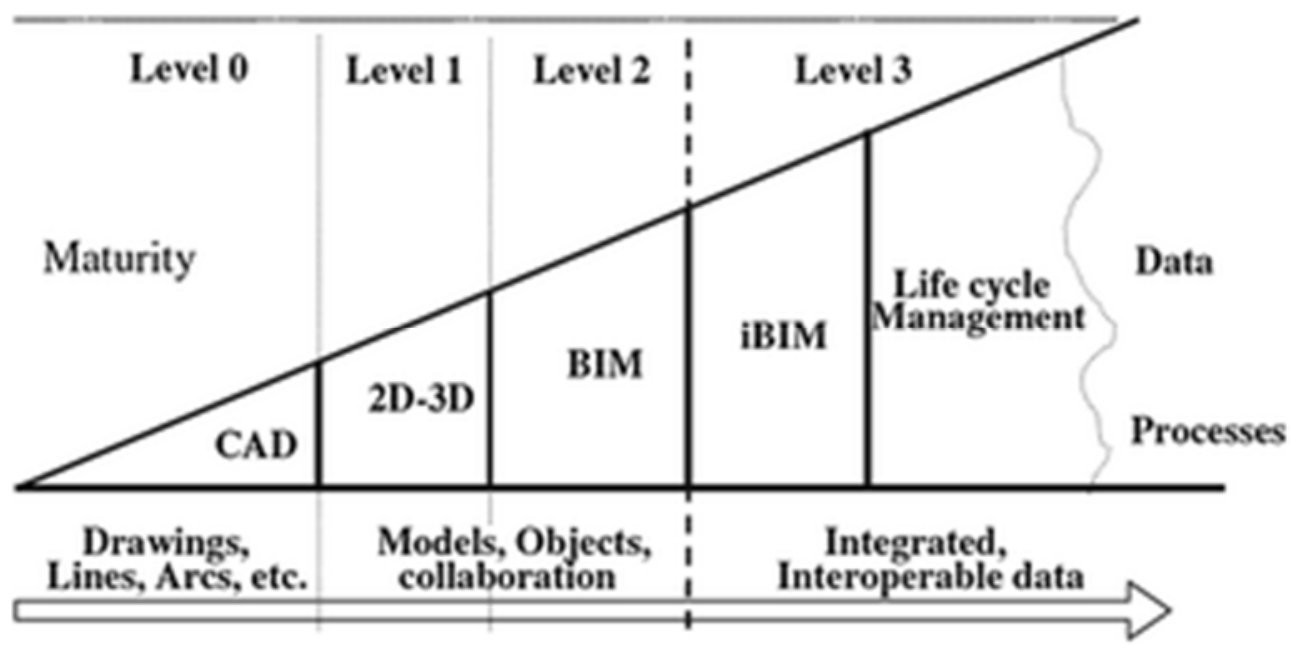

Figure 1: BIM maturity levels [16].

These maturity levels are subject to evaluation within companies and to do so, maturity models are mainly used. They allow companies to position themselves in relation to a level, according to their practices and resources. They are divided into several types. 


\subsection{Types of maturity model}

Maturity models are grouped into three main types, as shown in Table 1, because of their schematic format to target the objectives to be achieved and assign a meaningful value of the level of maturity [15].

Table 1: The three model types.

\begin{tabular}{|l|l|l|}
\hline Model types & Objectives for maturity assessment & Examples \\
\hline $\begin{array}{l}\text { Descriptive maturity } \\
\text { matrix }\end{array}$ & $\begin{array}{l}\text { Define and guide the implementation of good } \\
\text { practices to achieve objective (or aim towards) }\end{array}$ & $\begin{array}{l}\text { BIMCMM, } \\
\text { OBIMA, } \\
\text { BIMM }\end{array}$ \\
\hline $\begin{array}{l}\text { The questionnaire } \\
\text { with a Likert scale }\end{array}$ & $\begin{array}{l}\text { Assess the current situation of companies in a } \\
\text { certain context }\end{array}$ & ISO 9004 \\
\hline Cumulative model & $\begin{array}{l}\text { Assess a company's ability to achieve a } \\
\text { certain objective }\end{array}$ & CMM, CMMI \\
\hline
\end{tabular}

Cumulative models such as the CMM group together capabilities, called "Key Process Areas (KPA)", for each maturity level. These key areas are assimilated to generic performance and capabilities such as "risk analysis" or "requirements' development".

\subsection{The CMM (Capability Maturity Model)}

The CMM is divided into eleven criteria, each referring to areas of expertise and activities related to BIM. These eleven criteria are each assessed according to a ten-point scale, and each mark describes a specific state of the company for the criterion assessed. The 11 criteria are grouped in Table 2 below, adapted from Succar et al. [17] and Liebich [18].

The evaluator must therefore identify the level of the subject of the study at one of the criteria in order to determine its performance. It is important to note that all levels below the one assessed are considered acquired as well as the skills and tools put in place [19], [20].

Table 2: The eleven Key Process Areas.

\begin{tabular}{|l|}
\hline \multicolumn{1}{|c|}{ KPA } \\
\hline Data richness \\
\hline Graphical information \\
\hline Roles or disciplines \\
\hline Change management \\
\hline Business process \\
\hline Information accuracy \\
\hline Interoperability/IFC support \\
\hline Life cycle views \\
\hline Timeliness/response \\
\hline Delivery method \\
\hline Spatial capability \\
\hline
\end{tabular}




\subsection{The CSF (critical success factors)}

In the second part of the study, all areas of the CMM were linked to factors that can be identified and prioritized for work to improve the maturity level. They are the Critical Success Factors (CSF).

\subsection{The actions}

The third phase of the model's study is similar to concrete actions to be carried out in the context of the implementation and development of BIM levels. Each of the action is linked to a given critical success factor, and this, depending on the criterion linked to the factor and the consequences of the action [21].

\subsection{Relationship between CMM and CSF}

It is possible to validate a criterion of the model only by referring to the different CSF associated with it. It was also possible to classify the factors according to their importance but also their influence on the CMM. For greater consistency, with a view of implementing BIM, and developing it, some of the factors have not been retained.

This first observation enables to consider the evaluation of the initial maturity of a company, then to target the major issues allowing an evolution only from the exploitation of the links with the CSF.

The second link in the study, between factor and actions, takes into account the actions defined on the basis of the success factors. Knowing the points to be improved to perfect your use of BIM, it is essential to follow the recommendations and actions put in place to this end.

The general path of the study can then be mapped and read as follow: assessment of maturity within the company, examination of the link between the CMM criteria and CSF, then find the links between the factors and the associated actions that will have to be carried out in order to reach the desired level of maturity. The general path can also be mapped as presented in Fig. 2.

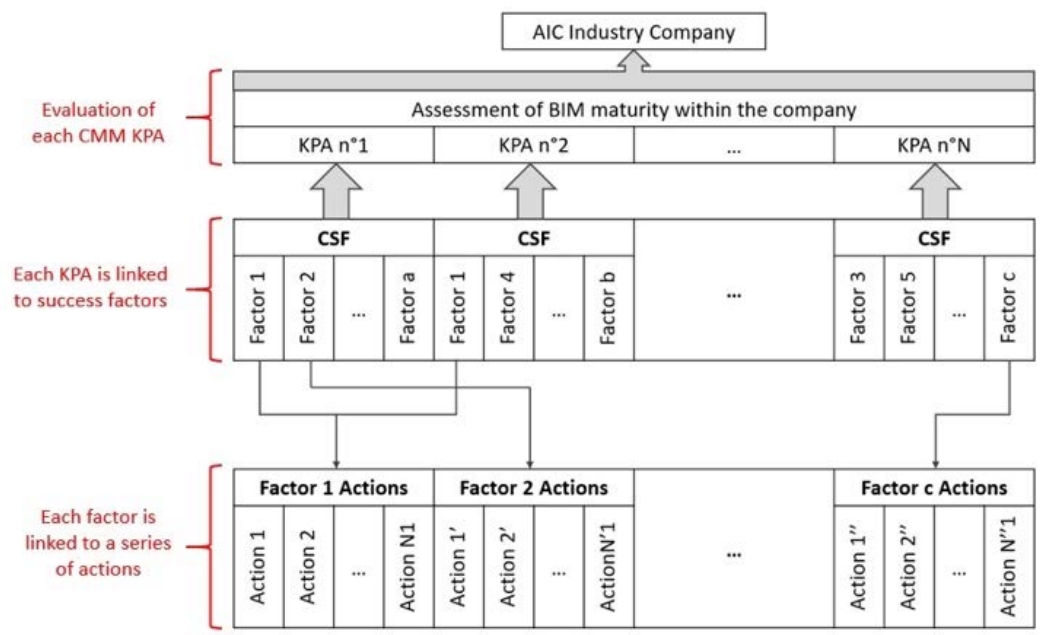

Figure 2: Level mapping. 
The actions proposed in the model do not constitute a precise and organized process along a timeline. These many actions can be perceived as levers that can be activated independently in order to improve one's control of the factors to which they are linked. The state of progress and control of BIM in AIC industry organizations is variable and does not allow to set up a generic plan based on a standardized initial maturity assessment. It is therefore more appropriate to carry out the actions described above in the development. Which will be adapted according to the needs defined by the company concerned. It is in this spirit that a survey was developed. It also aims at identifying the type of company that is interested in BIM, and their current practices if they already have them. Finally, this study allows us to know what the companies' expectations about BIM are.

Unlike its neighbour countries such as Spain, the United Kingdom, Germany and the Netherlands, France is significantly behind in the implementation of BIM into its industry. But, aware of this underdevelopment and so since the 2014 European directive stating that it is possible to require the use of BIM in public procurement (2014/24/EU-art.22.4), France is encouraging companies to comply with BIM.

The following survey reports on the implementation and current use of BIM in France.

The questionnaire is divided into three sections:

\section{THE SURVEY}

1. Identify the roles and types of companies of the interviewees

2. For companies using BIM, identify their use and target their expectations

3. For companies not using BIM, understand the reasons and motivations for not using it

For 1,150 requests sent, 104 responses were collected and analyzed, representing approximately $10 \%$ of the survey response rate. All respondents are employed in the construction industry.

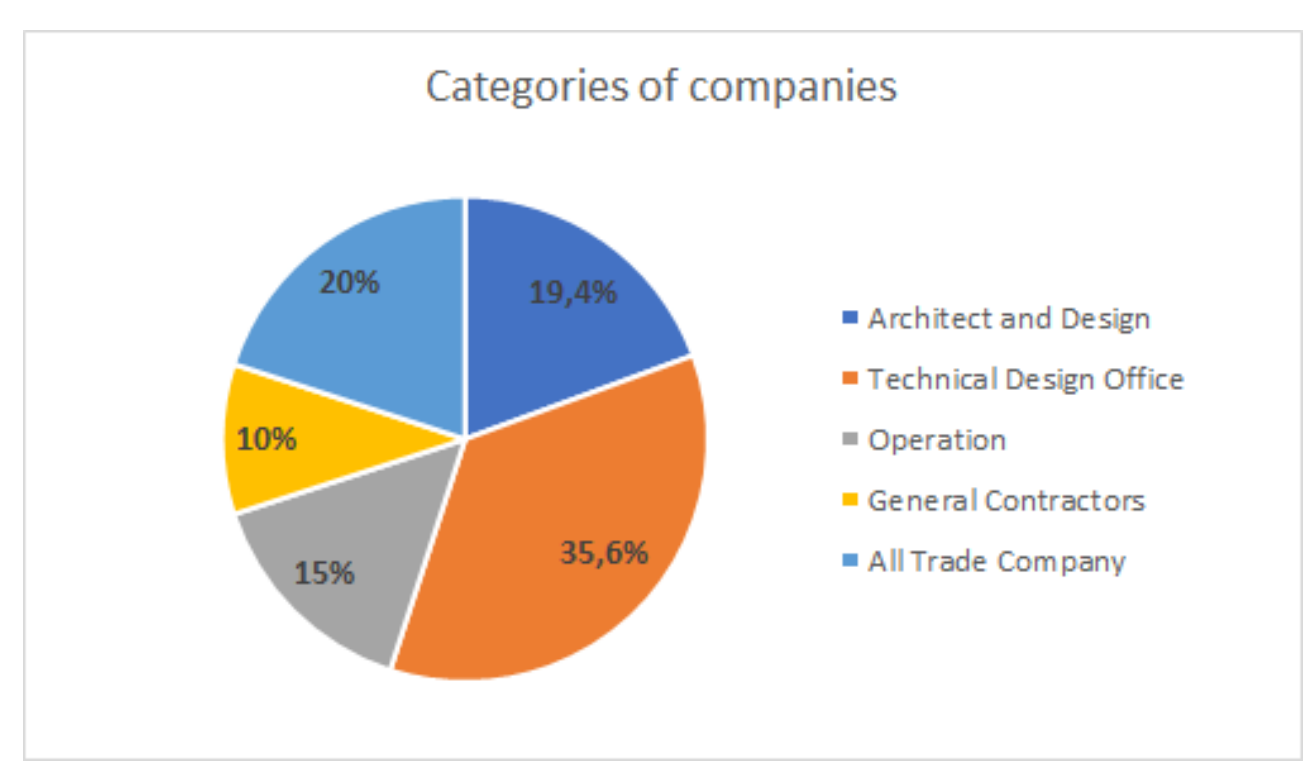

Figure 3: Categories of companies. 
As shown in Fig. 3, one third of the companies are ranked in the design office category (TDO), which reinforces the idea that most of the practices and expectations of companies are focused on technical issues (modelling, standardization, etc.) change and information management. In addition, the choice of tools for the operation of the BIM process is at the heart of considerations, in particular throughout the choice of exchange format and the company's needs.

The size of the companies is also to be taken into account for the change management component and also let assess the financial potential resources of each company. Fig. 4 below presents the distribution of firms by size.

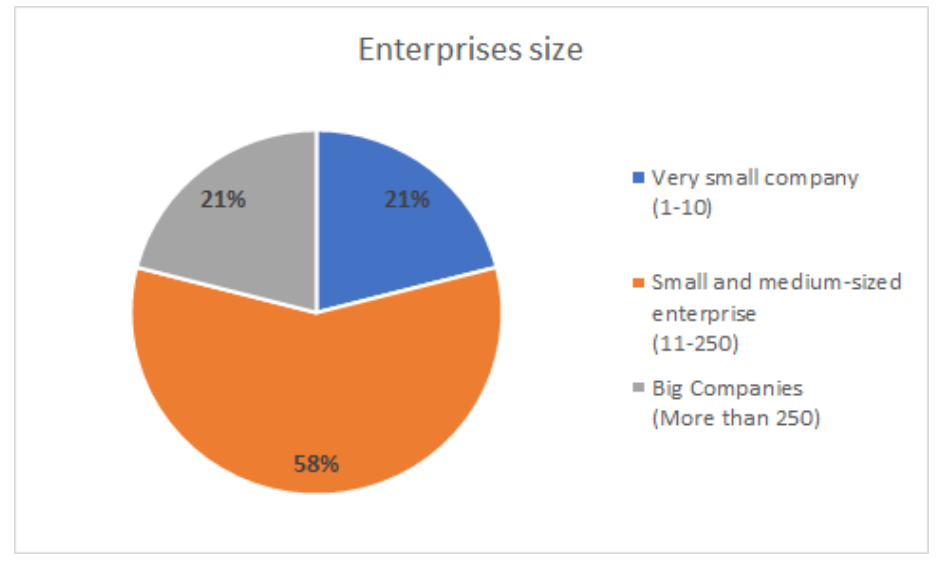

Figure 4: Companies size.

Looking at the results, we can see that $79 \%$ of the companies surveyed are in the TPE (very small company )/PME (small and medium-sized enterprise ) category. Some of these companies have just begun to integrate BIM into their practices and/or structure. The others have not yet started, due to the lack of funding, time, or motivation.

Based on the number of companies using BIM, the study has been divided into two parts. One section dedicated to companies using BIM and the other to those which are not using it yet.

\subsection{BIM insiders}

The main question of this survey dealt with how respondents assessed the current level of maturity of their companies. Let recall in the survey, the different maturity levels were defined as follows:

Level 1: We work with models (2D and 3D) that are not shared externally, by using CAD systems.

Level 2: We work in collaboration with other disciplines on models (3D, 4D and 5D) that we share between experts.

Level 3: We work collaboratively around a single, centralized model (4D, 5D and 6D) containing all the life cycle data of the construction.

The fist question regarding maturity levels was about the current level of the companies. The answers are grouped in Fig. 5. 


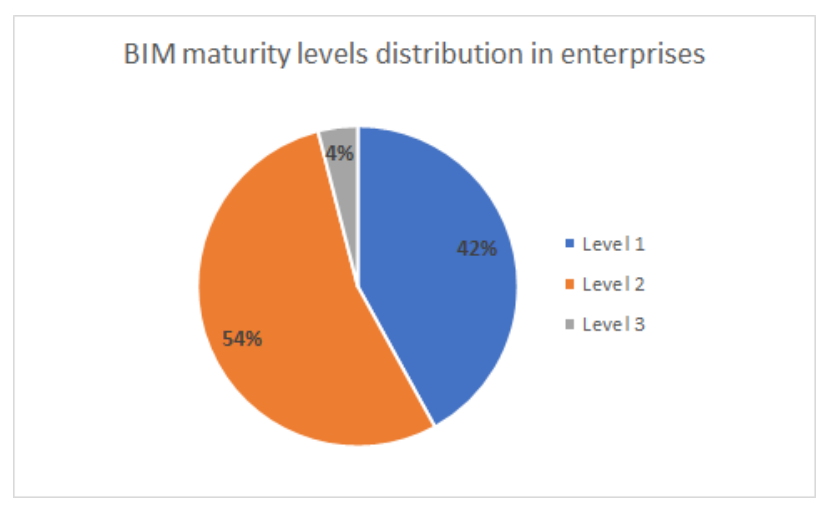

Figure 5: BIM maturity levels distribution in companies.

The results that came out from this question were predictable. Indeed, maturity level 2 is now the most widespread within AIC companies (even if it is often poorly exploited). It is not surprising that this response represents the largest percentage. The very low percentage for level 3 is not surprising either due to the fact that level 3 is still very difficult to access for small and medium-sized structures in the construction industry. The percentage of companies with maturity level 1 can dramatically change in different surveys. It just enables to get the maturity level of a company at a certain moment.

For three quarters of the companies surveyed, the use of BIM remains recent (less than four years). For a percentage of projects carried out in BIM of less than 50\%. These results, presented in Fig. 6, are in line with the various figures produced by the CSTB (Survey for the CSTB barometer, 2018) [22] within the framework of the PTNB. More than $68 \%$ of the companies asked use BIM as part of a strategic approach (focused on internal company skills) coupled with a contractual approach (in response to requirements defined in a BIM agreement or charter).

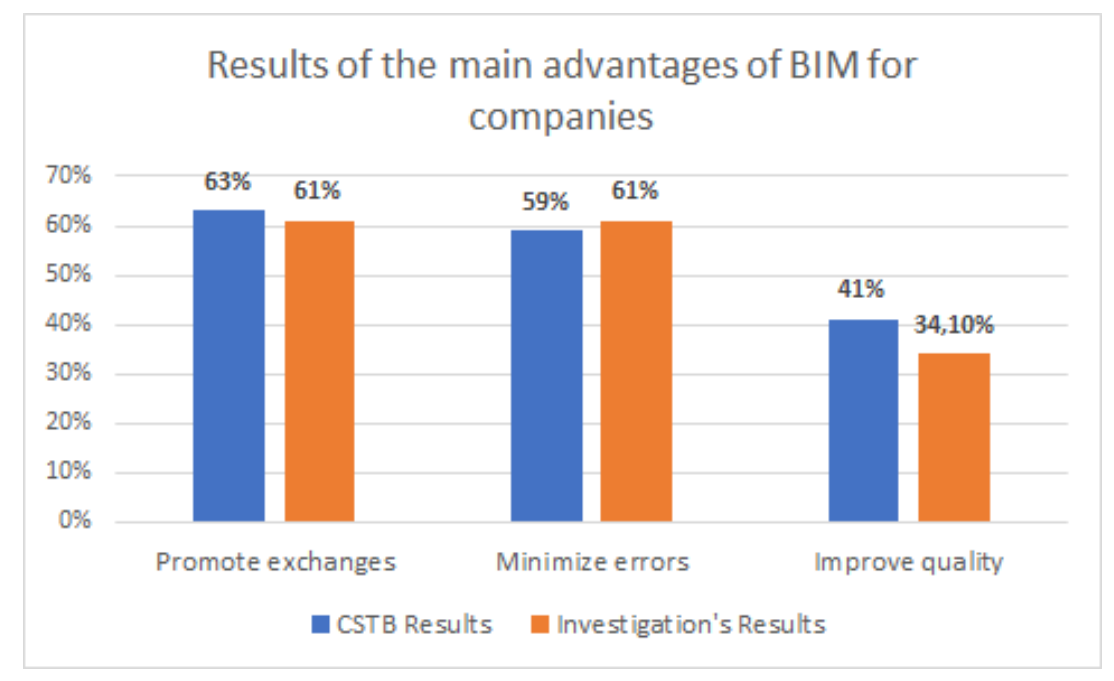

Figure 6: Advantages of BIM for companies [23]. 
After identifying the percentage of BIM use, it was important to identify current uses and future profits expected by companies. This is to correlate the different actions associated with the CFS defined in the model.

As with the question on the percentage of BIM used in firms, the results on the main benefits of BIM are equivalent to the survey conducted by the CSTB [22].

As explained above, another important aspect of the survey is to be able to target the future profits expected by companies when using BIM. All the most important of them are grouped in Fig. 7.

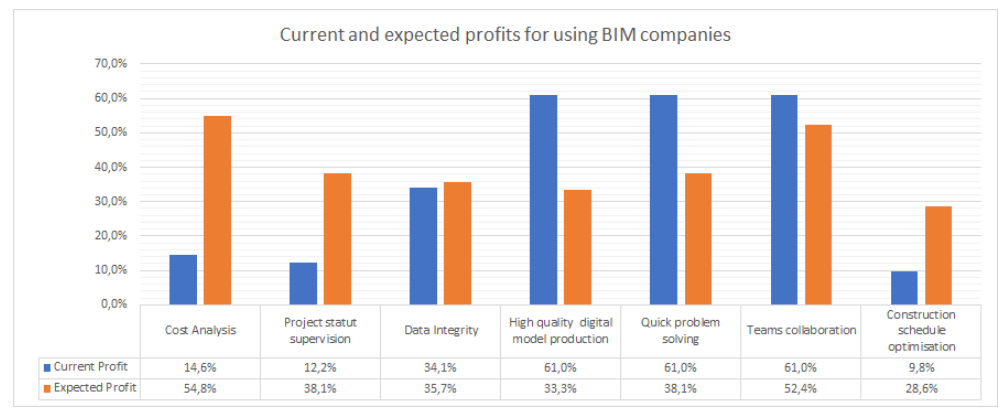

Figure 7: Benefits of BIM.

The three main benefits that emerge from this comparison are a better cost analysis $(+40.2 \%)$, a better control on the status of the project $(+25.9 \%)$ and the optimization of the construction planning $(+18.8 \%)$. These three benefits are accessible from the moment that the BIM is already implemented in these companies and that the company have already reached a level of maturity greater than or equal to level 2. Indeed, they allow the exploitation of the digital model and information beyond the three main dimensions of space.

These different responses have been linked to the actions associated with each criterion defined in the study to determine which actions were the most in demand.

\subsection{The uninitiated at BIM}

The second section of the survey focused exclusively on the questions related to potential technical and information management training and maturity audits. The first asking question was about their will of using BIM. The results are grouped in Fig. 8 below.

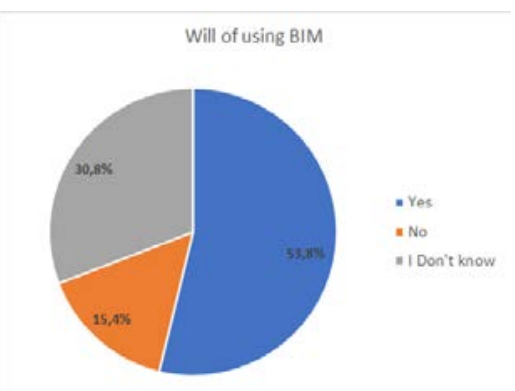

Figure 8: Will of using BIM. 
The share of undecided and negative responses is exactly equal as the number of people who have never heard of BIM. It is therefore legitimate to ask whether this reluctance to introduce BIM is not due to some form of fear of change, even if it is also very likely to result from a financial constraint or simply from a lack of time.

Companies were then asked if they would like to be trained in the use of modelling software and the implementation of BIM processes. Moreover, these companies have been asked if they were interested in identifying their maturity level. The results are presented in Fig. 9 below.

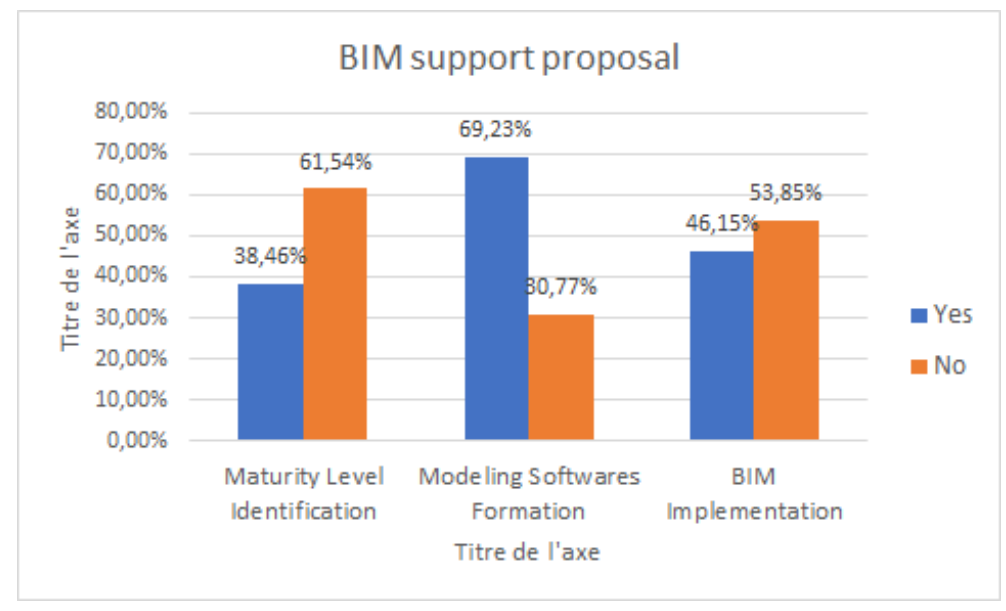

Figure 9: BIM support proposal.

\subsection{Projection after analysis}

The development is reaching the objective set at the beginning of the study, namely, the development of a structured methodology to support construction professionals in the implementation and evolution of BIM through the concept of maturity. The result presented throughout the study is based on numerous hypotheses and deductions that can be considered subjective or even arbitrary for some. This is what makes possible to highlight the limits of this model, limits that can be studied in order to overcome them and thus reinforce the current model, which will be more relevant.

One of the first aspects that can be pointed out is the fact that at present, very few procedures for the full implementation of BIM are available in the literature. This lack of a reference framework represents one of the first difficulties for construction companies today to implement and use BIM effectively. Consequently, a comprehensive description of a BIM implementation methodology would be a major step forward with significant benefits for the entire sector. The path established during this study does not aim to meet this need. The actions that compose it are more to be put on the account of common sense and simply brings a development axis as for the challenges of the implementation of BIM. Moreover, the actions are not ranked in terms of importance or even chronology. Thus, one of the additional contributions for the study would be to classify and develop the actions in order to obtain an action plan specific to each factor. In addition, classifying actions according to their difficulty in implementation and listing the limits of non-validation of actions can be considered. 
The model focuses essentially on activities. A second area for improvement would be to gradually include the notion of roles that could provide real relevant information for the company on how to organize its approach and allocate its resources in the most optimal way possible. Finally, if we go beyond the strict framework imposed by the issues related to BIM, it is still possible to progress in the maturity levels of the latter. The addition of a legal dimension seems essential. In fact, this dimension constitutes a major challenge for companies in the construction sector since it has a direct impact on the functioning of BIM within the structure but also on the applications that can be made of it. However, at present no standardization has been put in place by the competent authorities, which implies that an update of this critical factor will have to be introduced in the future. This area of expertise being quite far from my skills, this issue was not taken into account when defining the success factors.

\section{CONCLUSION}

The issues related to the implementation of BIM and support in the evolution of maturity levels have been at the centre of previous research. Interoperability between construction stakeholders through information management, building lifecycle management and change management are major benefits that can be achieved thanks to the use of BIM. These benefits include installation, learning its methodologies and its optimization at all levels of the organization.

The small number of publications on BIM implementation issues is directly related to the low level of development of this subject, which is based solely on theoretical and not empirical cases. However, the interest of the various construction stakeholders in this subject remains very strong, particularly because of the development of new technologies and the benefits observed in our European and North American neighbours who master BIM and use it to its full potential. From these observations the following objectives appeared to capitalize and transcode the information in the literature and then establish a process for evaluating the maturity of BIM within companies based on specific criteria related to critical success factors that would themselves be validated through concrete actions.

The links between the various components of the maturity model developed let introduce the notion of BIM implementation to support in maturity levels. These links also make possible to establish a survey based on the expectations of BIM users, which was one of the major elements of the study, both in terms of investment and reflection, and in terms of benefits for the exploitation of the process. This survey has also provided an opportunity to discuss the relevance of the model developed.

The study of the survey results validated the relevance of most of the established actions and the critical success factors associated with them. The many comments and suggestions made by the survey respondents also allow to question the value of certain criteria in order to optimize the consistency of the model.

The final process, closely linked to the literature, offers many significant contributions to the origin of construction professionals, whether or not they use BIM. It describes the different steps to follow and the criteria to validate in order to improve your command of BIM. This improvement is possible throughout the implementation of concrete actions to provide as much information and assistance as possible to BIM users.

However, this process is not yet a viable and infallible tool that would be fully usable by the AIC industry. Indeed, certain limits in the process have been identified: tests must be carried out and allow the criteria to be weighted according to the actions carried out or not. Other limitations related to the process itself suggest areas for improvement. For example, the creation of a chronological application plan for the implementation and evolution of 
maturity within the company would be a significant contribution in an industry that is currently very litigious. Taking into account more success factors and therefore associated actions is an evolution that must be considered in order to expand the model database. Consideration of the legal aspect related to BIM is also a possible development but will only be really possible when a standardisation of BIM practices will be established by the competent authorities.

BIM remains a recent tool in the construction industry and is still very misunderstood by AIC industry players today. This misunderstanding stems in particular from practices associated with BIM that reflect current standards that seem deeply rooted in a reluctance to change. However, as with any major technological advance, some companies at the forefront have already experienced this process and adapted their methods after a successful implementation. Though, scientific research remains the most qualified today (in partnership with BIM users) to provide significant support to the AIC industry. It could lead to the improvement of processes such as the one described during the study and its standardization could lead to a major advance in the use and understanding of BIM.

\section{REFERENCES}

[1] Joblot, L., Contribution à la mise en oeuvre du BIM en rénovation: Proposition d'un Modèle de Maturité BIM spécifique. PhD thesis report, 2018.

[2] Dakhil, A., Alshawi, M. \& Underwood, J., BIM client maturity, literature review. Proceedings of the 12th International Post-Graduate Research Conference, University of Salford, pp. 229-238, 2015.

[3] Babic, G. \& Rebolj, D., Culture change in construction industry from 2D toward BIM based construction. Journal of Information Technology in Construction, 21, pp. 8699, 2016.

[4] Eastman, J.K. \& Eastman, K.L., Perception of status consumption and the economy. Journal of Business and Economic Research, 9(7), pp. 9-20, 2011.

[5] Tranchant, A., Beladjine, D. \& Beddiar, K., BIM in French SMEs: From innovation to necessity. WIT Transactions on The Built Environment, vol. 169, WIT Press: Southampton and Boston, pp. 135-142, 2017.

[6] Eastm, J.K., Goldsmith, R.E. \& Flynn, L.R., Status consumption in consumer behaviour: scale development and validation. Journal of Marketing Theory and Practice, 7(3), pp. 41-52, 1999.

[7] Delcambre Bertrand 2014, Rapport Mission Numérique Batiment. Ateliers du Numérique, 2015.

[8] European Commission, Micro Small and Medium Sized Enterprises: Definition and Scope, 2003.

[9] Sanchez, A. et al., BIM benefits realisation management. Delivering Value with BIM. A Whole of Life Approach, pp. 58-81, 2016.

[10] Miettinen, R. \& Paavola, S., Beyond the BIM utopia: Approaches to the development and implementation of building information modelling. Automation in Construction, 43, pp. 84-91, 2014.

[11] Wu, C., Xu, B., Mao, C. \& Li, X., Overview of BIM maturity measurement tools. Journal of Information Technology in Construction, 22, pp. 34-62, 2017.

[12] Volk, R., Stengel, J. \& Schultmann, F., Building information modelling for existing building: Literature review and future needs. Automation in Construction, 38, pp. 109127, 2014.

[13] Azzouz, A., Copping, A., Shepherd, P. \& Duncan, A., Using the ARUP BIM maturity measure to demonstrate BIM implementation in practice. Proceedings of the 32nd 
Annual ARCOM Conference, eds. P.W. Chan \& C.J. Neilson. Manchester: Association of Researchers in Construction Management, 1, pp. 25-34, 2016.

[14] Succar, B. \& Kassem, M., Macro-BIM adoption: Conceptual structures. Automation in Construction, 57, pp. 64-79, 2015.

[15] Farruck, C., Fraser, P. \& Gregory, M., Development of a structured approach to assessing practice in product development collaborations. Proceedings of the Institution of Mechanical Engineers, 217(8), pp. 1131-1144, 2003.

[16] Dos Santos, S., Vendrametto, O. \& Correia, C.F., Profile of Building Information Modeling (BIM) Tools Maturity in Brazilian Civil Construction Scenery, Published in APMS, 2015.

[17] Succar, B., Sher, W. \& Williams, A., Measuring BIM performance: Five metrics. Architectural Engineering and Design Management, 8, pp. 120-142, 2012.

[18] Liebich, T., IFC $2 x$ Edition 3 Model Implementation Guide, Building SMART International Modeling Support Group, 2009

[19] Tsai, M.-H., Mom, M. \& Hsieh, S.-H., Developing critical success factors for the assessment of BIM technology adoption, Part I, methodology and survey. Journal of the Chinese Institute of Engineers, 37, pp. 845-858, 2014.

[20] Ramanayaka, C.D. \& Venkatachalam, S., Reflection on BIM development practices at the pre-maturity. Procedia Engineering, 123, pp. 462-470, 2015.

[21] Smith, D.K. \& Tardif, M., Building Information Modelling: A Strategic Implementation Guide for Architects, Engineers, Constructors and Real Estate Asset Managers, 2009.

[22] Survey for the CSTB barometer, Supporting the Stakeholders of Construction Activity Report, pp. 38-47, 2015.

[23] Survey for the CSTB barometer, Le BIM dans le secteur du BTP: Résultats de la 3e enquête réalisée pour le baromètre du PTNB sur l'utilisation des outils numériques et digitaux par les professionnels du Bâtiment, 2018. 